\title{
The role of the mitochondrial calcium uniporter in cerebral ischemia/reperfusion injury in rats involves regulation of mitochondrial energy metabolism
}

\author{
QIN ZHAO, SHILEI WANG, YU LI, PENG WANG, SHUHONG LI, YUNLIANG GUO and RUYONG YAO \\ Department of Anesthesiology, Affiliated Hospital of Qingdao University Medical College, \\ Qingdao, Shandong 266000, P.R. China
}

Received November 25, 2012; Accepted February 5, 2013

DOI: $10.3892 / \mathrm{mmr} .2013 .1321$

\begin{abstract}
The mitochondrial calcium uniporter (MCU) maintains intracellular $\mathrm{Ca}^{2+}$ homeostasis by transporting $\mathrm{Ca}^{2+}$ from the cell cytosol into the mitochondrial matrix and is important for shaping $\mathrm{Ca}^{2+}$ signals and the activation of programmed cell death. Inhibition of MCU by ruthenium red (RR) or Ru360 has previously been reported to protect against neuronal death. The aim of the present study was to analyze the mechanisms underlying the effects of MCU activity in a rat model of cerebral ischemia/reperfusion (I/R) injury. Adult male Wistar rats were divided into 4 groups; sham, I/R, I/R + RR and I/R + spermine (Sper) and were subjected to reversible middle cerebral artery occlusion for $2 \mathrm{~h}$ followed by $24 \mathrm{~h}$ of reperfusion. A bolus injection of RR administered 30 min prior to ischemia was found to significantly decrease the total infarct volume and reduce neuronal damage and cell apoptosis compared with ischemia/reperfusion values. However, treatment with Sper, an activator of the MCU, increased the injury induced by I/R. Analysis of energy metabolism revealed that I/R induced progressive inhibition of complexes I-IV of the electron transport chain, decreased ATP production, dissipated the mitochondrial membrane potential and increased the generation of reactive oxygen species. Treatment with RR ameliorated the condition, while spermine had the opposite effect. In conclusion, blocking MCU was demonstrated to exert protective effects against I/R injury and this process may be mediated by the prevention of energy failure.
\end{abstract}

Correspondence to: Professor Shilei Wang, Department of Anesthesiology, Affiliated Hospital of Qingdao University Medical College, Wutaishan Road, Huangdao, Qingdao, Shandong 266000, P.R. China

E-mail: wshlei@yahoo.com.cn

Key words: ischemia/reperfusion injury, mitochondrial calcium uniporter, energy metabolism

\section{Introduction}

Ischemic stroke is a worldwide public health issue. Ischemic stroke causes brain dysfunction and is one of the most frequent causes of mortality. The most effective strategy for treating the injury and limiting infarct size is the early restoration of coronary blood flow to the ischemic myocardium. However, this treatment is often associated with functional and structural damage during reperfusion (1), which leads to cerebral edema, brain hemorrhage and even neuronal death. Among the animal models, middle cerebral artery occlusion (MCAO) followed by reperfusion is most frequently used as the animal model of focal cerebral ischemia and resembles human ischemic stroke (2). The molecular mechanism of ischemic brain injury is not completely understood. Mitochondria have been demonstrated to be involved in the regulation of the apoptosis process and are important for ischemic cell death. Mitochondria are the source of energy for sustaining life and in addition to their role as energy-producing organelles, they participate in the majority of physiological processes, including the cell cycle, the production of reactive oxygen species (ROS), apoptosis and ion balance $(3,4)$. Significant progress has been made in previous years to understand the mechanisms of mitochondrial biology.

Mitochondrial channels, whose activities are linked to a large number of mitochondrial functions and pathologies, have received significant attention (5). Over 50 years ago, using a variety of techniques under experimental conditions, the mitochondrial calcium uniporter (MCU) was identified to be responsible for the uptake of $\mathrm{Ca}^{2+}$ by mitochondria, driven by the large electrical potential difference between the cytosol and the mitochondrial matrix. Previous studies have demonstrated that under pathological conditions, including ischemia/reperfusion ( $\mathrm{I} / \mathrm{R})$ injury, mitochondria accumulate significant amounts of $\mathrm{Ca}^{2+}$ from the cytosol via MCU. Increases in mitochondrial $\mathrm{Ca}^{2+}$ concentration $\left(\left[\mathrm{Ca}^{2+}\right]_{\mathrm{m}}\right)$ induce the opening of the mitochondrial permeability transition pore (MPTP) leading to inhibition of ATP synthesis, increased ROS production, cytochrome $c$ release and cell death by apoptosis (6,7). Ruthenium red (RR) and Ru360 block the MCU to reduce the $\mathrm{Ca}^{2+}$ influx and, therefore, exert a beneficial effect during I/R injury by preventing $\mathrm{Ca}^{2+}$ accumulation (8). 
Oxidative phosphorylation, involving the electron transport chain (ETC) and ATP synthase, provides the vast majority of cellular energy and drives all cellular processes. The ETC is composed of NADH dehydrogenase (complex I), succinate dehydrogenase (complex II), cytochrome $c$ reductase (complex III) and cytochrome $c$ oxidase (complex IV). Previous studies have revealed that under pathological conditions, including I/R injury, the ETC causes cell damage and even triggers death processes due to depressed ATP production and the generation of ROS (9). ROS are produced via the ETC, however, these chemically reactive molecules also damage electron transport complexes (10), leading to further respiratory dysfunction and increases in ROS production, which results in a positive feedback cycle during I/R. Appropriate treatment strategies may be administered to break this cycle and protect mitochondrial structure and function.

Our previous studies have revealed that ROS production is reduced by inhibiting MCU (11). Thus, the current study was based on the hypothesis that electron transport complexes and cerebral infarction area are affected by the activity of the MCU in ischemic/reperfused rats. ATP levels, ROS production, changes in mitochondrial membrane potential $\left(\Delta \Psi_{\mathrm{m}}\right)$ and HE and TUNEL staining results were analyzed to to determine the mechanisms underlying the effects of MCU activity in a rat model of cerebral I/R injury.

\section{Materials and methods}

Chemicals and reagents. RR, spermine and rhodamine 123 were purchased from Sigma-Aldrich (St Louis, MO, USA). The ATP assay kit was purchased from Beyotime Institute of Biotechnology (Jiangsu, China). Mitochondria isolation and assay kits for mitochondrial complex studies were purchased from Genmed Scientifics Inc. (Shanghai, China). The Bradford protein assay kit was purchased from Nanjing Jiancheng Bioengineering Institute (Nanjing, China).

Animals. Male Wistar rats weighing 250-300 g (supplied by the experimental animal center of Qingdao Drug Inspection Institute, Qingdao, China) were randomly divided into 4 groups of 12 animals: I (sham), without coronary artery ligation, underwent identical surgical procedures as the $\mathrm{I} / \mathrm{R}$ group; II (I/R), received saline solution $(0.9 \%) 30 \mathrm{~min}$ prior to MCAO, i.e., ischemia was induced for $2 \mathrm{~h}$ followed by $24 \mathrm{~h}$ of reperfusion; III (I/R + RR), received a bolus injection of $\mathrm{RR}$ dissolved in saline solution $30 \mathrm{~min}$ prior to MCAO; IV (I/R + Sper), received a bolus injection of spermine dissolved in saline solution $30 \mathrm{~min}$ prior to MCAO.

MCAO model. MCAO rats were generated as described previously (12). Briefly, rats were anesthetized with $10 \%$ chloral hydrate $(350 \mathrm{mg} / \mathrm{kg}$, i.p.). The right common carotid artery (CCA), internal carotid artery (ICA) and external carotid artery (ECA) were exposed via a midline incision on the neck. Next, the CCA and ECA were ligated (near the bifurcation) with 4-0 surgical sutures and the ICA was clipped with an artery clip. After a small incision was made in the CCA, a nylon filament with a diameter of $0.285 \mathrm{~mm}$ was introduced into the ICA (18-20 $\mathrm{mm}$ from the external-internal carotid artery bifurcation) through the CCA. The nylon filament was maintained in position for $2 \mathrm{~h}$ and was then carefully removed to restore the blood flow. During surgery, body temperature was maintained at $36.5-37.5^{\circ} \mathrm{C}$ using a heating pad.

Neurological deficit scoring evaluation. Neurological deficits were evaluated following $2 \mathrm{~h}$ ischemia and $24 \mathrm{~h}$ reperfusion according a scale system described previously (13): 0, normal, no neurological deficit signs; 1 , failure to extend contralateral forepaw on lifting of the animal by the tail; 2 , circling to the contralateral side, but normal posture at rest; 3 , falling to the contralateral side at rest; 4 , no spontaneous locomotor activity. The model was confirmed at scores 2-4.

Measurement of infarct volumes. To analyze the infarct volumes, TTC (2,3,5-triphenyltetrazolium chloride) staining was used. Rats were sacrificed $24 \mathrm{~h}$ following reperfusion, brains were removed rapidly and frozen at $-20^{\circ} \mathrm{C}$ for $5 \mathrm{~min}$. The brains were sectioned into $2-\mathrm{mm}$ thick coronal slices using a brain-sectioning block and stained with standard $1 \%$ TTC for $15 \mathrm{~min}$ at $37^{\circ} \mathrm{C}$. Images of sections were captured and analyzed using the Image-Pro Plus 5.1 analysis system (Media Cybernetics, Rockville, MD, USA). Infarct volume (\%HLV) was calculated using the following equation: $\% \mathrm{HLV}=\{$ [total infarct volume - (right hemisphere volume - left hemisphere volume)]/left hemisphere volume\} x 100.

HE staining. Following reperfusion $(24 \mathrm{~h})$, rats were anesthetized and perfused with $200 \mathrm{ml} 0.9 \%$ sodium chloride, followed by $100 \mathrm{ml} 4 \%$ paraformaldehyde (PFA) in $0.1 \mathrm{M}$ phosphate-buffered saline (PBS, pH 7.4) through the left ventricle. Brains were removed and fixed in 4\% PFA overnight. Sections (3-mm thick) cut coronally from optic chiasma to occipital pole were embedded in paraffin and cut into several segments $(4-\mu \mathrm{m}$ thick). Coronal sections were stained with hematoxylin-eosin. Sections were also used for TUNEL staining.

TUNEL staining. An in situ cell death detection kit (Roche Diagnostics GmbH, Mannheim, Germany) was used to detect apoptotic cell death. According to the manufacturer's instructions with minor modifications, paraffin embedded sections were deparaffinized and rehydrated by graded ethanol, followed by treatment with proteinase $\mathrm{K}$ for $15 \mathrm{~min}$ and $3 \%$ $\mathrm{H}_{2} \mathrm{O}_{2}$ for $10 \mathrm{~min}$ at room temperature. Following three $10 \mathrm{~min}$ washes in PBS, sections were incubated with terminal deoxynucleotidyl transferase at $37^{\circ} \mathrm{C}$ for $2 \mathrm{~h}$. Sections were washed in PBS three times for 10 min each and further incubated with anti-digoxigenin conjugate for $30 \mathrm{~min}$ at $37^{\circ} \mathrm{C}$. Following washing, 3,3'-diaminobenzidine was used to visualize apoptotic cells. Images were captured at a magnification of $\mathrm{x} 400$ using the Nikon ECLIPSE TE300 fluorescence microscope (Nikon Instruments, Inc., Melville, NY, USA).

Isolation ofmitochondria. Brain mitochondria were isolated by differential centrifugation, using the Functional Mitochondria Isolation kit (Genmed Scientifics, Inc., Wilmington, DE, USA). All procedures were performed on ice to maintain the temperature at $4^{\circ} \mathrm{C}$. Rats were anesthetized, decapitated and the perifocal penumbra zone of the brain was immediately removed. Tissue was immediately placed in ice-cold PBS to remove impurities, then processed into fine pieces. The 


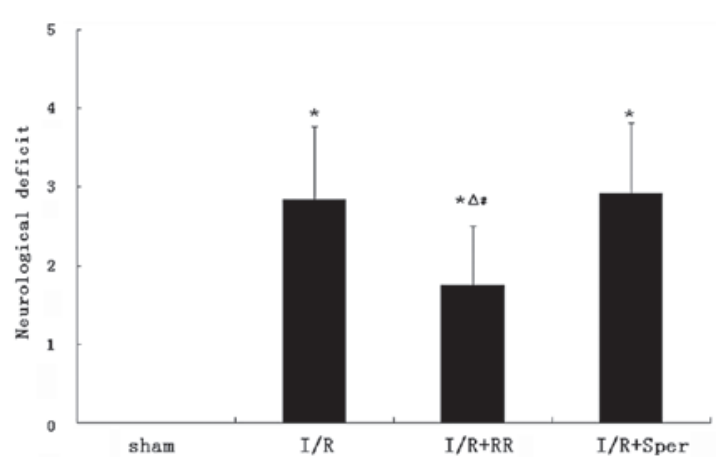

Figure 1. Effect of mitochondrial calcium uniporter activity on neurological deficits. Neurological deficit scores were significantly increased in the I/R and $\mathrm{I} / \mathrm{R}+$ Sper groups compared with the sham group, but no significant differences were observed between the two groups. Neurological deficit scores were significantly reduced in the $\mathrm{I} / \mathrm{R}+\mathrm{RR}$ group compared with the I/R group. ${ }^{*} \mathrm{P}<0.01$, vs. sham; ${ }^{\wedge} \mathrm{P}<0.01$, vs. I/R; ${ }^{*} \mathrm{P}<0.01$, vs. I/R + Sper. Each group, $n=12$. I/R, ischemia/reperfusion; $R R$, ruthenium red; Sper, spermine.

tissue was homogenized in isolation medium using a glass homogenizer. The homogenate was centrifuged at $1,000 \mathrm{xg}$ for $10 \mathrm{~min}$. The supernatant was retained and centrifuged at $10,000 \mathrm{x} \mathrm{g}$ for $10 \mathrm{~min}$. The resulting pellet was resuspended and rinsed by centrifuging at $10,000 \mathrm{x} \mathrm{g}$ for $5 \mathrm{~min}$. The final mitochondrial pellet was resuspended in reaction buffer and stored at $-80^{\circ} \mathrm{C}$. Protein concentration was determined with the Bradford protein assay using BSA as a standard (14).

Measurement of mitochondrial complex activity. Mitochondrial freeze-thaw cycles were repeated 3 times to enable enzyme release. The activities of mitochondrial complexes I-IV were measured using an assay kit according to the manufacturer's instructions and a microplate reader (Tecan Group Ltd., Männedorf, Switzerland).

Measurements of intracellular ATP levels. ATP levels were determined using the ATP detection kit (Beyotime Institute of Biotechnology) following the luciferin-luciferase method (15). Briefly, tissue was homogenized with a glass homogenizer in a lysis buffer from the ATP detection kit, followed by centrifuging at $12,000 \mathrm{x} \mathrm{g}$ for $5 \mathrm{~min}$ at $4^{\circ} \mathrm{C}$. The supernatant was retained for the ATP test. Supernatant or standard buffer (both $100 \mu \mathrm{l}$ ) were mixed with $100 \mu \mathrm{l}$ ATP detection working dilution on a black plate and luminescence was measured immediately. Standard curves were generated, to which the ATP level was referred and the protein concentration of each treatment group was determined using the Bradford protein assay.

Measurement of mitochondrial membrane potential. Flow cytometry was used to monitor $\Delta \Psi_{\mathrm{m}}$ according to the method described previously (16) with minor modifications. Rhodamine 123, a fluorescent cationic dye, uses a transmembrane potential-dependent mechanism to enter the mitochondria. The tissue was digested with pancreatic enzymes and made into suspended cells, which were washed with PBS. In the dark, cells were loaded with Rhodamine 123 (final concentration, $5 \mu \mathrm{mol} / \mathrm{l}$ ) at $37^{\circ} \mathrm{C}$ for $30 \mathrm{~min}(17)$ and then washed twice with PBS. Following incubation, fluorescence was determined using flow cytometry (FC500 MPL; Beckman Coulter, Miami, FL, USA) at an excitation wavelength of
$488 \mathrm{~nm}$ and emission wavelength of $530 \mathrm{~nm}$. Data were analyzed using the CXP 2.1 software package.

Measurement of intracellular ROS generation. 2',7'-Dihydrodichlorofluorescein diacetate (DCFH-DA), a membrane-permeable probe, was used to evaluate ROS generation (18). The non-fluorescent dye freely diffuses through the cell membrane and is hydrolyzed to the nonfluorescent DCFH by intracellular esterases. When DCFH is oxidized by ROS, it yields the fluorescent product, DCF. A sample was incubated with DCFH-DA $(100 \mu \mathrm{M})$ in the dark at $37^{\circ} \mathrm{C}$ for $20 \mathrm{~min}$ and DCF fluorescence intensity was measured using a fluorescence spectrophotometer (Tecan Group Ltd.) at an excitation wavelength of $488 \mathrm{~nm}$ and an emission wavelength of $525 \mathrm{~nm}$.

Statistical analysis. SPSS 17.0 statistical software was used to perform statistical analysis. $\mathrm{P}<0.05$ was considered to indicate a statistically significant difference. All data are expressed as mean \pm SD. ANOVA was used to compare the difference between groups.

\section{Results}

Role of the MCU in neurological deficits following I/R injury. Following reperfusion $(24 \mathrm{~h})$, rats in the sham group exhibited no neurological deficits. Neurological deficit scores were observed to be significantly higher in the I/R and I/R + Sper groups than in the sham group $(\mathrm{P}<0.01)$, however, no significant differences were observed between the I/R and I/R + Sper groups. By contrast, scores were significantly lower in the $\mathrm{I} / \mathrm{R}+\mathrm{RR}$ group $(\mathrm{P}<0.01)$ than in the $\mathrm{I} / \mathrm{R}$ group (Fig. 1).

Role of the $M C U$ in cerebral infarct volume. Infarct volume measurements revealed that rats from the I/R group exhibited a larger infarct area than the rats from the sham group, in which no infarction was observed. In the I/R group, irregular pale areas supplied by the middle cerebral artery were observed in sections. Treatment with RR $(2.5 \mathrm{mg} / \mathrm{kg})$ in the $\mathrm{I} / \mathrm{R}+\mathrm{RR}$ group

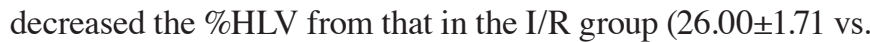
$35.43 \pm 0.74 \%$, respectively; $\mathrm{P}<0.01)$. Spermine $(5 \mathrm{mg} / \mathrm{kg})$ in the $\mathrm{I} / \mathrm{R}+$ Sper group increased the \%HLV $(36.57 \pm 1.31 \%)$, however, the change in infarct volume from that in brains that underwent I/R only was not determined to be significant (Fig. 2).

Role of the MCU in neuronal damage and cell apoptosis of brain tissue. HE staining was used to evaluate the histopathological values at $24 \mathrm{~h}$ following reperfusion. No brain infarction was found in the sham group and the cell outline was clear, the structure was compact and the nucleolus was clearly visible. Cells in the I/R and I/R + Sper groups were arranged sparsely and revealed pyknotic nuclei. In addition, cell outlines were undefined, structures were disordered and deformation of cells was severe. In the I/R + RR group, there were fewer necrotic cells, cell outlines were relatively clear and cell structures were compact (Fig. 3A).

The TUNEL assay was used to determine cell apoptosis. TUNEL-positive cells with apoptotic bodies and dark staining were considered to be apoptotic cells (Fig. 3A). Apoptotic cells were almost unobservable in the sham group $(6.50 \pm 0.85 \%)$. The numbers of TUNEL-positive cells in the I/R and I/R + Sper 
A

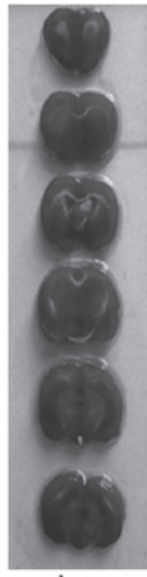

sham

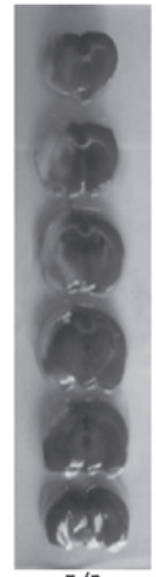

$\mathrm{I} / \mathrm{R}$

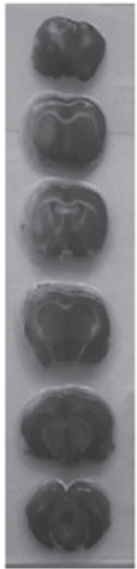

$\mathrm{I} / \mathrm{R}+\mathrm{RR}$

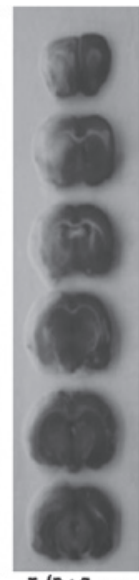

$\mathrm{I} / \mathrm{R}+\mathrm{Sper}$
B

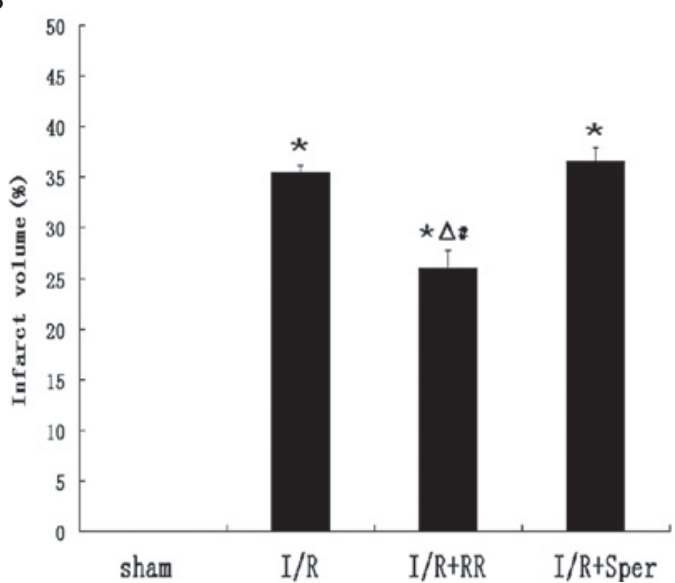

Figure 2. Effect of mitochondrial calcium uniporter activity on cerebral infarct volume. \%HLV was significantly increased in the $\mathrm{I} / \mathrm{R}$ and $\mathrm{I} / \mathrm{R}+\mathrm{Sper}$ groups compared with that in the sham group, but no significant difference was found between the two groups. \%HLV was significantly lower in the I/R $+\mathrm{RR}$ group than in the I/R group. ${ }^{~} \mathrm{P}<0.01$, vs. sham; ${ }^{\circ} \mathrm{P}<0.01$, vs. I/R; ${ }^{\prime \prime} \mathrm{P}<0.01$, vs. $\mathrm{I} / \mathrm{R}+$ Sper. Each group, $\mathrm{n}=3 . \% \mathrm{HLV}$, infarct volume; $\mathrm{I} / \mathrm{R}$, ischemia/reperfusion; $\mathrm{RR}$, ruthenium red; Sper, spermine.

A
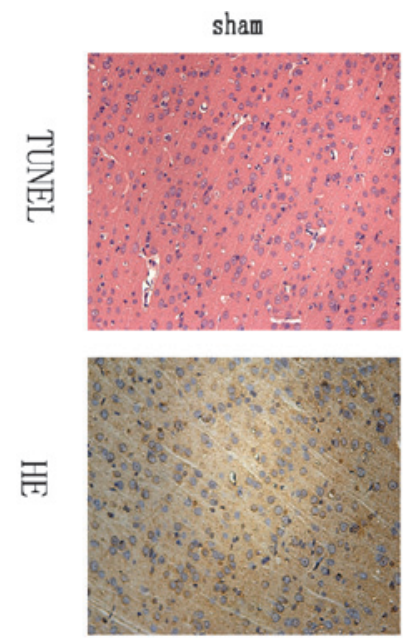

I/R
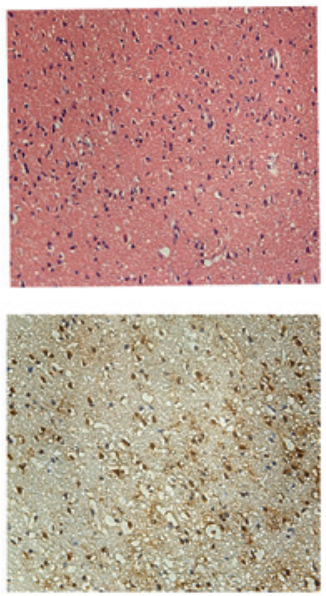
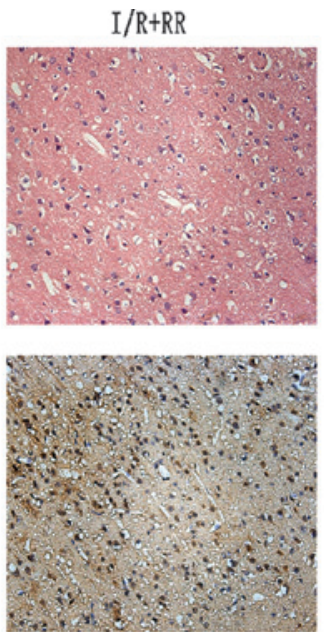
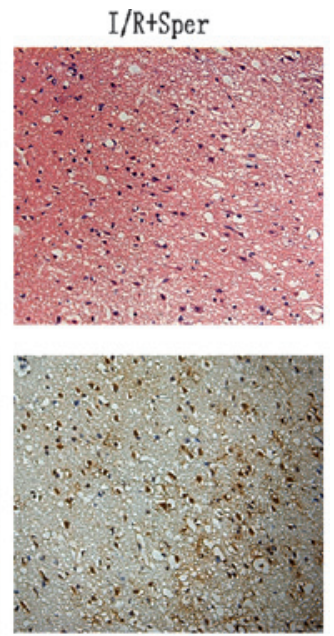

B

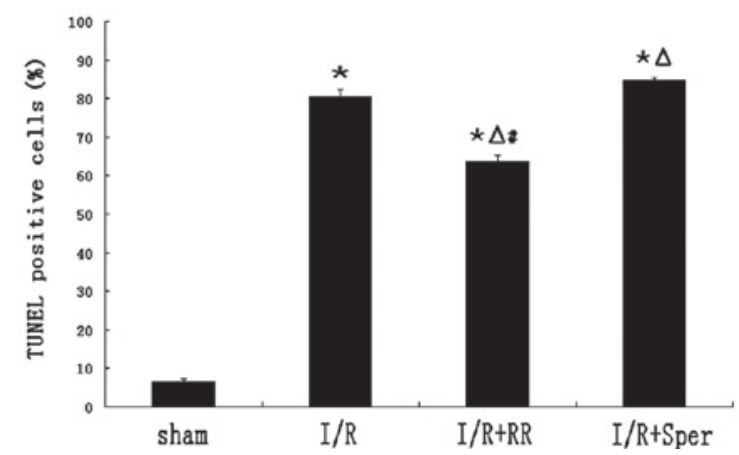

Figure 3. Effect of mitochondrial calcium uniporter activity on neuronal damage and cell apoptosis. (A) HE and TUNEL staining for neurons in sham, I/R, $\mathrm{I} / \mathrm{R}+\mathrm{RR}$ and $\mathrm{I} / \mathrm{R}+$ Sper groups were assessed at $24 \mathrm{~h}$ following reperfusion. (B) The proportion of TUNEL-positive cells increased markedly in the I/R group compared with that in the sham group. RR treatment significantly reduced the number of TUNEL-positive cells compared with that in the I/R group. Following treatment with spermine, the number of TUNEL-positive cells increased significantly. ${ }^{*} \mathrm{P}<0.01$, vs. sham; ${ }^{\wedge} \mathrm{P}<0.01$, vs. I/R; ${ }^{*} \mathrm{P}<0.01$, vs. $\mathrm{I} / \mathrm{R}+\mathrm{Sper}$. Each group, $\mathrm{n}=3$. I/R, ischemia/reperfusion; RR, ruthenium red; Sper, spermine.

groups were higher than those in the sham rats $(\mathrm{P}<0.01)$ and were slightly higher in the I/R + Sper group than in the I/R group. The number of TUNEL-positive cells was significantly lower in the I/R + RR group $(63.63 \pm 1.60 \%)$ than in the I/R group, indicating that RR treatment significantly ameliorated cell survival and inhibited apoptosis (Fig. 3B).
Effect of MCU on mitochondrial complex activities. To evaluate the effect of MCU activity on mitochondrial energy metabolism, the enzyme activities of complexes I-IV were measured in mitochondria isolated from brains. Activities of complexes I, II, III and IV were demonstrated to be decreased by $\sim 40,43,59$ and $51 \%$, respectively, in animals suffering 


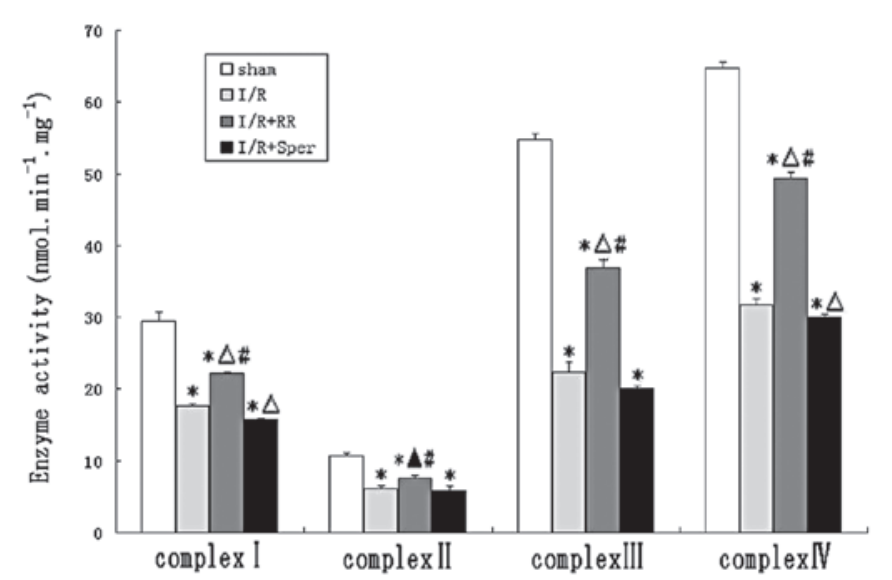

Figure 4. Effect of mitochondrial calcium uniporter activity on the activities of ETC complexes following I/R. Activities of ETC complexes decreased significantly in the $\mathrm{I} / \mathrm{R}$ and $\mathrm{I} / \mathrm{R}+$ Sper groups compared with those in the sham group. The activities of complexes I-IV in the I/R + RR group increased significantly. ${ }^{*} \mathrm{P}<0.01$, vs. sham; ${ }^{\wedge} \mathrm{P}<0.01$, vs. I/R; ${ }^{\wedge} \mathrm{P}<0.05$, vs. I/R; ${ }^{*} \mathrm{P}<0.01$, vs. I/R + Sper. Each group, $n=3$. I/R, ischemia/reperfusion; RR, ruthenium red; Sper, spermine; ETC, electron transport chain.

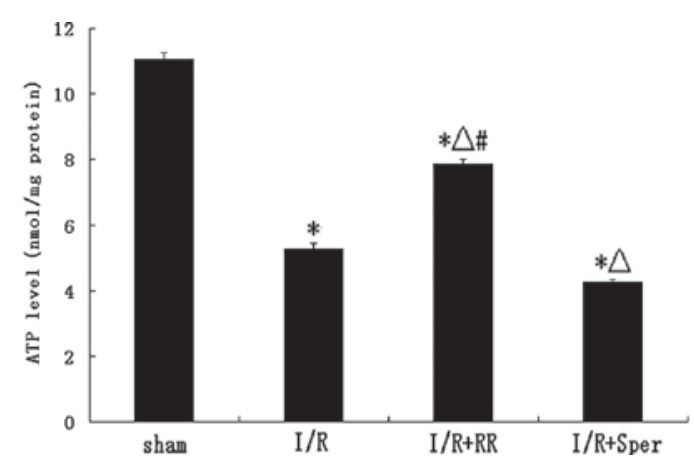

Figure 5. Effect of mitochondrial calcium uniporter activity on intracellular ATP generation. ATP levels in the I/R and I/R + Sper groups decreased compared with those in the sham group and differences between the two groups were observed. Following treatment with RR, ATP levels increased significantly compared with those in the I/R group. ${ }^{*} \mathrm{P}<0.01$, vs. sham; ${ }^{\circ} \mathrm{P}<0.01$, vs. $\mathrm{I} / \mathrm{R} ;{ }^{\#} \mathrm{P}<0.01$, vs. I/R + Sper. Each group, $\mathrm{n}=3$. I/R, ischemia/reperfusion; RR, ruthenium red; Sper, spermine.

from I/R and increased by $\sim 15,12,26$ and $27 \%$, respectively, following RR treatment. The activities of the ETC complexes were lower in the $I / R$ group than in the sham group $(\mathrm{P}<0.01)$. The results indicate that $\mathrm{I} / \mathrm{R}$ led to inhibition of mitochondrial complexes. RR treatment significantly ameliorated the activities of complexes I, III and IV $(\mathrm{P}<0.01)$ and complex II $(\mathrm{P}<0.05)$, while complex I, II, III and IV activities decreased in the $\mathrm{I} / \mathrm{R}+$ Sper group (Fig. 4).

Effect of MCU on ATP levels. Fig. 5 demonstrates that ATP levels in I/R rats decreased following $24 \mathrm{~h}$ reperfusion. ATP levels in the RR-treated group were $\sim 51 \%$ higher than in the I/R group $(\mathrm{P}<0.01)$, whereas, following treatment with spermine, ATP levels were lower than in the I/R group $(\mathrm{P}<0.01$; Fig. 5$)$.

Effect of MCU on mitochondrial membrane potential. The mitochondrial membrane potential has been widely used to study mitochondrial health. Rhodamine 123, a mitochondrion-selective fluorescent dye which is rapidly sequestered by
$\mathbf{A}$
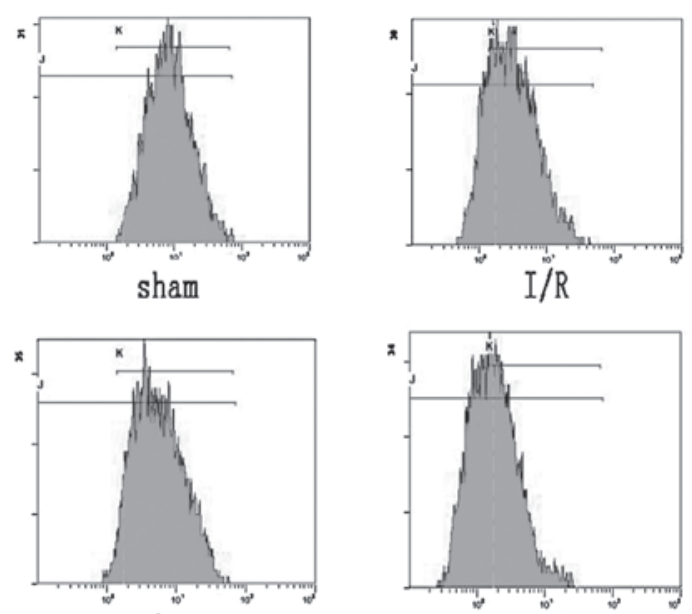

$\mathrm{I} / \mathrm{R}+\mathrm{RR}$

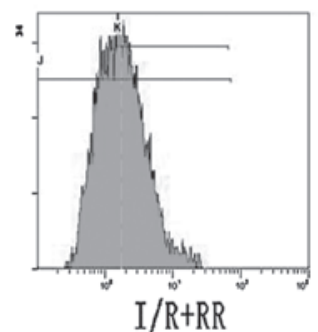

B

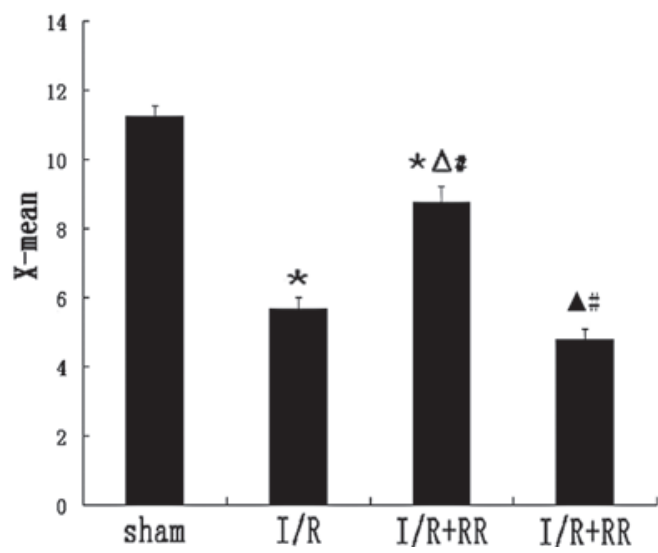

Figure 6. Effect of mitochondrial calcium uniporter activity on $\Delta \Psi_{\mathrm{m}}$ (A) FCM analysis of mitochondrial membrane potential of neurons in each group. (B) Comparison of $\mathrm{X}$-mean in the 4 groups. " $\mathrm{P}<0.01$, vs. sham; ${ }^{\wedge} \mathrm{P}<0.01$, vs. I/R; ${ }^{\wedge} \mathrm{P}<0.05$, vs. I/R; ${ }^{*} \mathrm{P}<0.01$, vs. I/R + Sper. Each group, $\mathrm{n}=3$. $\Delta \Psi_{\mathrm{m}}$, change in mitochondrial membrane potential; $\mathrm{I} / \mathrm{R}$, ischemia/reperfusion; RR, ruthenium red; Sper, spermine.

mitochondria, was used as a marker of membrane disruption. The X-mean was found to be significantly lower in I/R cells $(5.67 \pm 0.32 ; \mathrm{P}<0.01)$ than in those of the sham group (Fig. 6B), with the wave moving left (Fig. 6A), indicative of a significant loss of membrane potential. RR treatment increased the $\mathrm{X}$-mean significantly $(8.76 \pm 0.45 ; \mathrm{P}<0.01)$, with the wave moving right compared with the $\mathrm{I} / \mathrm{R}$ group. However, the wave did not reach normal levels (sham group). RR treatment attenuated the dissipation of mitochondrial membrane potential caused by reperfusion injury. In the presence of spermine to induce MCU opening, the X-mean increase was higher than in the I/R group $(4.80 \pm 0.30 ; \mathrm{P}<0.05)$, indicating mitochondrial depolarization (Fig. 6).

Effect of MCU activity on intracellular ROS generation. To observe whether ROS generation is affected by MCU activation, ROS generation was monitored by detection of DCFH-DA fluorescence. In the sham group, low levels of DCFH-DA fluorescence were observed. There was a marked increase of fluorescence in the I/R group, indicative of an increase in ROS production. Following treatment with RR, ROS generation was observed to decrease significantly $(\mathrm{P}<0.01)$. By contrast, as revealed in Fig. 7, fluorescence in the I/R + Sper group 


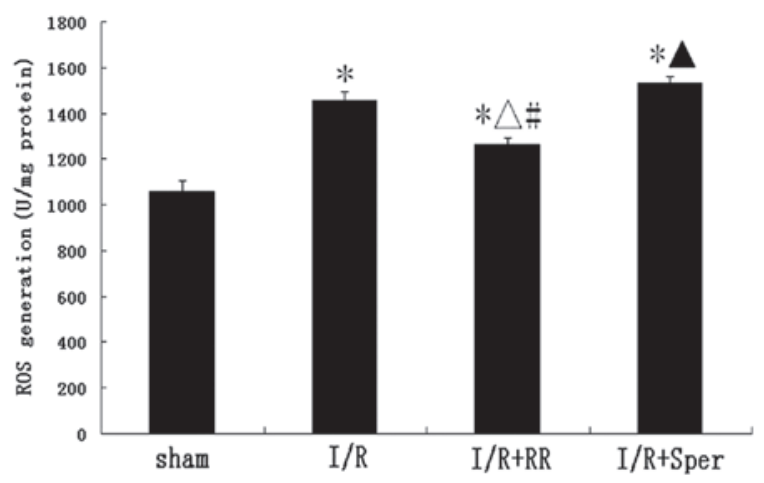

Figure 7. Effect of mitochondrial calcium uniporter activity on ROS generation. RR treatment significantly decreased mitochondrial ROS generation induced by ischemia/reperfusion (I/R). ROS generation in the $\mathrm{I} / \mathrm{R}+$ Sper group was higher than that in the I/R group. ${ }^{*} \mathrm{P}<0.01$, vs. sham; ${ }^{\wedge} \mathrm{P}<0.01$, vs. $\mathrm{I} / \mathrm{R} ;{ }^{\wedge} \mathrm{P}<0.05$, vs. I/R; ${ }^{\mathrm{P}} \mathrm{P}<0.01$, vs. I/R + Sper. Each group, $\mathrm{n}=3$. RR, ruthenium red; ROS, reactive oxygen species.

increased markedly and was higher than that in the I/R group $(\mathrm{P}<0.05)$. In the present study, MCU activity was demonstrated to regulate intracellular ROS generation.

\section{Discussion}

Ischemic stroke is one of the leading causes of mortality and disability in the world. However, at present, only a limited number of therapeutic strategies have been found to target ischemic brain injury and it remains a major public health issue. MCAO followed by reperfusion has been widely used to study ischemia mechanisms and potential interventions. In the present study, MCU inhibition was found to have anti-apoptotic and neuroprotective effects during cerebral I/R injury in rats. The results revealed that treatment with $\mathrm{RR}$, which blocks MCU, significantly ameliorated neurological deficit scores and led to a significant reduction in cerebral infarction during MCAO. HE staining revealed that RR significantly reduced neuronal injury. In addition, RR was observed to exhibit neuroprotective effects against ischemia-induced apoptosis of neuronal cells. RR also demonstrated protective effects against reperfusion injury in the heart $(19,20)$. By contrast, treatment with spermine, an activator of the uniporter, led to converse results. MCU was demonstrated to have a regulatory effect in focal cerebral I/R injury, consistent with previous studies (21-23). In addition, the mechanism by which MCU regulates cerebral I/R injury was hypothesized to be associated with improved mitochondrial energy metabolism due to MCU inhibition.

Mitochondria play a crucial role in the production of energy and are the site of the majority of ATP generation. Decreases in cellular ATP levels, impaired mitochondrial oxidative respiration and large influxes of $\mathrm{Ca}^{2+}$ that result in cellular excitotoxicity and apoptosis (24) are present during I/R. The inhibition of key mitochondrial respiratory complexes has been observed to be the cause of ischemia-induced mitochondrial dysfunction (25). Cells are almost entirely dependent on mitochondrially generated ATP for their energy and, therefore, a defect at any level of the mitochondrial oxidative phosphorylation machinery has profound effects on brain function. As a key event in I/R injury, mitochondrial $\mathrm{Ca}^{2+}$ overload does not always result in MPTP opening and cytochrome $c$ release to detrimentally affect mitochondrial function. Overload is known to lead to inhibition of mitochondrial respiratory complexes, with subsequent enhancement of ROS generation, which then results in the inhibition of respiratory complexes and mitochondrial dysfunction.

The MCU, which has been investigated for $\sim 40$ years, is a highly selective ion channel located in the mitochondrial inner membrane. It is responsible for the uptake of $\mathrm{Ca}^{2+}$ in mitochondria driven by the large electrical potential difference between the cytosol and mitochondrial matrix (26). $\mathrm{Ca}^{2+}$ transport activity is inhibited by RR and its associated compound, RuR360 and is also modulated by aliphatic polyamines, including spermine and aminoglycosides. Although MCU has recieved considerable attention for decades, this gated channel has not been cloned or isolated and its molecular identity remains controversial. Uncoupling protein (UCP) 2 and UCP3 were previously reported to be the essential components of the uniporter machinery (27), however, the results remain controversial and further studies are required to definitively elucidate the molecular identity of the channels. In a previous study, a $54 \mathrm{kDa}$ protein known as mitochondrial calcium uptake 1 was identified, whose silencing regulates mitochondrial $\mathrm{Ca}^{2+}$ uptake (28). As a single-pass transmembrane protein, it is unlikely to function as a $\mathrm{Ca}^{2+}$ channel. Instead, it is likely to act as a fundamental subunit of the complex for the uptake machinery. In 2011, MCU was identified by analyzing 14 genes in detail and concluded that the protein Ccdc109A may be a component of MCU $(29,30)$. A subsequent study by these authors hypothesized that MCU is an inner mitochondrial membrane protein with the C-terminus facing the intermembrane space $(29,30)$.

Mitochondrial $\mathrm{Ca}^{2+}$ is key to the regulation of mitochondrial functions, ranging from mediating signaling pathways between the cytosol and the mitochondrial matrix to modulating mitochondrial energy metabolism. However, the molecular mechanisms underlying mitochondrial $\mathrm{Ca}^{2+}$ transport remain unclear (31). Within the mitochondrial matrix, enhanced $\mathrm{Ca}^{2+}$ levels improve electron transport and increase NADH and ATP production (32). Therefore, mitochondrial ATP output is altered to meet cellular ATP demands and, under specific conditions, reduce ROS formation (33). However, under pathological conditions, including I/R, $\mathrm{Ca}^{2+}$ accumulation in mitochondria has been proposed to be significant in cellular injury (34). ROS increase the cytosolic $\mathrm{Ca}^{2+}$ concentration, affecting the sarcoplasmic reticulum and sarcolemmal membranes during I/R (35), and then increases $\left[\mathrm{Ca}^{2+}\right]_{\mathrm{m}}(20)$. The overload in mitochondrial $\mathrm{Ca}^{2+}$, together with cyclophilin D, induce MPTP opening (36), which releases cytochrome $c$, Smac/DIABLO and apoptosis-inducing factors involved in apoptotic death signaling (37). In addition, mitochondrial $\mathrm{Ca}^{2+}$ overload triggers the generation of factors, including ROS and free fatty acids (38), and also promotes MPTP opening. There are several mechanisms that are hypothesized to be involved in $\mathrm{Ca}^{2+}$-induced $\mathrm{ROS}$ production. Mitochondrial complexes are known to be damaged when animals are subjected to $I / R$, causing an excessive release of ROS. Under physiological conditions, $\mathrm{Ca}^{2+}$ may be a partial inhibitor of the ETC, leading to ROS production. Respiratory chain enzymes, NADH dehydrogenase (complex I) and 
cytochrome $c$ oxidase (complex IV), participate in energy metabolism and the generation of ROS and are vulnerable to ROS attack. Intracellular ATP production is reported to be the prime target of free radicals in hypoxia/ischemia (39). NADH dehydrogenase and cytochrome c oxidase activities were observed to decrease following I/R, which may be the direct or indirect result of ROS damage in the penumbra zone. Treatment with RR has been reported to protect against the loss of complex I activity in isolated cardiomyocytes subjected to hypoxia/reoxygenation (40), therefore, it has been hypothesized that mitochondrial $\mathrm{Ca}^{2+}$ uptake during reoxygenation is involved in the mechanism. A number of previous studies have reported that inhibition of complex I, in combination with $\mathrm{Ca}^{2+}$ overload, leads to enhanced ROS generation in in vitro and in vivo systems $(41,42)$. Under physiological conditions, $\mathrm{Ca}^{2+}$ also dissociates cytochrome $c$ from the mitochondrial inner membrane by triggering MPTP opening and cytochrome $c$ blocks the respiratory chain at complex III effectively, causing an increase in ROS production $(43,44)$. The spectra of cytochromes a/a3 is reported to be altered by $\mathrm{Ca}^{2+}$ in isolated complex IV (45). Furthermore, $\mathrm{Ca}^{2+}$ stimulation of the tricarboxylic acid (TCA) cycle increases the metabolic rate, which results in increased respiratory chain electron leakage. $\mathrm{Ca}^{2+}$ stimulation of nitric oxide synthase (NOS), which generates $\mathrm{NO}$, inhibits the respiratory chain at complex IV (46) and subsequently enhances ROS generation from the Q cycle. NO demonstrates a marked contribution to changes in mitochondrial energy metabolism during the I/R transition. In addition, $\mathrm{NO}$ and $\mathrm{Ca}^{2+}$ are known to inhibit complex I together and may also increase ROS generation by this complex $(47,48)$.

In this study, ROS production was observed to be increased in animals who had undergone $\mathrm{I} / \mathrm{R}$, but was attenuated by the MCU inhibitor, RR, which permeates slowly into the cell and specifically inhibits mitochondrial $\mathrm{Ca}^{2+}$ uptake. This observation is consistent with a previous study which reported that RR decreased ROS production, induced by $\mathrm{Ca}^{2+}$ overload (47). Ru360, a specific MCU inhibitor, was previously identified to be important in the modulation of ROS under conditions involving excessive mitochondrial $\mathrm{Ca}^{2+}$ overload (49). In the present study, activation of the uniporter with spermine ( $5 \mathrm{mg} / \mathrm{kg}$ ) caused the levels of ROS production to increase, whereas RR caused them to decrease, indicating that MCU activities are important for the regulation of ROS production during brain I/R injury. In addition, analysis of the respiratory chain demonstrated an improvement in I/R-related mitochondrial respiratory complex I, II, III and IV dysfunction with RR treatment, with the opposite results following spermine treatment. However, the underlying mechanisms remain to be elucidated. MCU has been previously hypothesized to affect mitochondrial respiratory complexes via regulation of mitochondrial $\mathrm{Ca}^{2+}$ and/or ROS. In addition, RR protection of mitochondrial respiratory complexes I-IV may increase ATP production, which is extremely important for the preservation of ATP-dependent cellular processes in I/R. These hypotheses are consistent with results of the present study. $\Delta \Psi_{\mathrm{m}}$ is known to be a pivotal factor for the determination of cellular survival during I/R. It has previously been reported that $\Delta \Psi_{\mathrm{m}}$ hyperpolarization is associated with the generation of ROS which, at specific levels, may trigger mitochondrial membrane depolarization (50). A previous study reported that $\Delta \Psi_{\mathrm{m}}$ decrease was an initiator and was also a consequence of MPTP opening (51). As as an inhibitor of MCU, Ru360 may normalize mitochondrial membrane depolarization by preventing the opening of the MPTP during I/R (22). The results of the present study revealed changes in $\Delta \Psi_{\mathrm{m}}$, specifically, membrane depolarization during I/R, normalization by RR and accelerated collapse following spermine treatment, that are indicative of the regulatory functions of MCU on $\Delta \Psi_{\mathrm{m}}$ during I/R.

In conclusion, the results of the present study indicate that MCU activities are important in I/R by regulating mitochondrial energy metabolism. Blocking the uniporter with RR increases the functional recovery of brains subjected to $I / R$ and the mechanism may be associated with improved energy metabolism. These results are likely to contribute to the understanding of MCU in I/R, however, additional studies must be performed to further elucidate the mechanism. In addition, the identification of novel effective drugs targeting the MCU for use in reperfusion therapy is likely to be of significant interest.

\section{References}

1. Jennings RB, Sommers HM, Smyth GA, Flack HA and Linn H: Myocardial necrosis induced by temporary occlusion of a coronary artery in the dog. Arch Pathol 70: 68-78, 1960.

2. Boyko M, Ohayon S, Goldsmith T, et al: Cell-free DNA - a marker to predict ischemic brain damage in a rat stroke experimental model. J Neurosurg Anesthesiol 23: 222-228, 2011.

3. Johnson DT, Harris RA, Blair PV and Balaban RS: Functional consequences of mitochondrial proteome heterogeneity. Am J Physiol Cell Physiol 292: C698-C707, 2007.

4. McBride HM, Neuspiel M and Wasiak S: Mitochondria: more than just a powerhouse. Curr Biol 16: R551-R560, 2006.

5. Sun Y, Deng T, Lu N, Yan M and Zheng X: B-type natriuretic peptide protect cardiomyocytes at reperfusion via mitochondrial calcium uniporter. Biomed Pharmacother 64: 170-176, 2010.

6. Sullivan PG, Rabchevsky AG, Waldmeier PC and Springer JE: Mitochondrial permeability transition in CNS trauma: cause or effect of neuronal cell death? J Neurosci Res 79: 231-239, 2005.

7. Demaurex N and Distelhorst C: Cell biology. Apoptosis - the calcium connection. Science 300: 65-67, 2003.

8. Yan Y, Liu J, Wei C, et al: Bidirectional regulation of $\mathrm{Ca}^{2+}$ sparks by mitochondria-derived reactive oxygen species in cardiac myocytes. Cardiovasc Res 77: 432-441, 2008.

9. Young TA, Cunningham CC and Bailey SM: Reactive oxygen species production by the mitochondrial respiratory chain in isolated rat hepatocytes and liver mitochondria studies using myxothiazol. Arch Biochem Biophys 405: 65-72, 2002.

10. Chen Q, Moghaddas S, Hoppel CL and Lesnefsky EJ: Ischemic defects in the electron transport chain increase the production of reactive oxygen species from isolated rat heart mitochondria. Am J Physiol Cell Physiol 294: C460-C466, 2008.

11. Yu N, Wang S, Wang P, et al: The calcium uniporter regulates the permeability transition pore in isolated cortical mitochondria. Neural Regen Res 72: 109-113, 2012.

12. Yang JP, Liu XF, Liu HJ, Xu GL and Ma YP: Extracellular signal-regulated kinase involved in NGF/VEGF-induced neuroprotective effect. Neurosci Lett 434: 212-217, 2008.

13. Xu X, Chua KW, Chua CC, et al: Synergistic protective effects of humanin and necrostatin-1 on hypoxia and ischemia/reperfusion injury. Brain Res 1355: 189-194, 2010.

14. Bradford MM: A rapid and sensitive method for the quantitation of microgram quantities of protein utilizing the principle of protein-dye binding. Anal Biochem 72: 248-254, 1976.

15. St John JB: Determination of ATP in Chlorella with the luciferin-luciferase enzyme system. Anal Biochem 37: 409-416, 1970.

16. Vander Heiden MG, Chandel NS, Williamson EK, et al: Bcl-xL regulates the membrane potential and volume homeostasis of mitochondria. Cell 91: 627-637, 1997.

17. Emaus RK, Grunwald R and Lemasters JJ: Rhodamine 123 as a probe of transmembrane potential in isolated rat-liver mitochondria: spectral and metabolic properties. Biochim Biophys Acta 850: 436-448, 1986. 
18. LeBel CP, Ischiropoulos $\mathrm{H}$ and Bondy SC: Evaluation of the probe 2',7'-dichlorofluorescin as an indicator of reactive oxygen species formation and oxidative stress. Chem Res Toxicol 5: 227-231, 1992.

19. Ferrari R, di Lisa F, Raddino R and Visioli O: The effects of ruthenium red on mitochondrial function during post-ischaemic reperfusion. J Mol Cell Cardiol 14: 737-740, 1982.

20. Miyamae M, Camacho SA, Weiner MVV and Figueredo VM: Attenuation of postischaemic reperfusion injury is related to prevention of $\left[\mathrm{Ca}^{2+}\right]_{\mathrm{m}}$ overload in rat hearts. Am J Physiol 271: H2145-H2153, 1996.

21. Griffiths EJ: Mitochondrial calcium transport in the heart: Physiological and pathological roles. J Mol Cell Cardiol 46: 789-803, 2009

22. García-Rivas Gde J, Carvajal K, Correa F and Zazueta C: Ru360, a specific mitochondrial calcium uptake inhibitor, improves cardiac post-ischaemic functional recovery in rats in vivo. $\mathrm{Br} \mathrm{J}$ Pharmacol 149: 829-837, 2006.

23. Clements-Jewery H: Mitochondria, the calcium uniporter and reperfusion-induced ventricular fibrillation. Br J Pharmacol 149: 811-813, 2006.

24. Rashidian J, Iyirhiaro GO and Park DS: Cell cycle machinery and stroke. Biochim Biophys Acta 1772: 484-493, 2007.

25. Almeida A, Allen KL, Bates TE and Clark JB: Effect of reperfusion following cerebral ischaemia on the activity of the mitochondrial respiratory chain in the gerbil brain. J Neurochem 65: 1698-1703, 1995.

26. Bernardi P: Mitochondrial transport of cations: channels, exchangers and permeability transition. Physiol Rev 79: 1127-1155, 1999.

27. Trenker M, Malli R, Fertschai I, Levak-Frank S and Graier WF: Uncoupling proteins 2 and 3 are fundamental for mitochondrial $\mathrm{Ca}^{2+}$ uniport. Nat Cell Biol 9: 445-452, 2007.

28. Perocchi F, Gohil VM, Girgis HS, et al: MICU1 encodes a mitochondrial EF hand protein required for $\mathrm{Ca}^{2+}$ uptake. Nature 467: 291-296, 2010.

29. De Stefani D, Raffaello A, Teardo E, Szabo I and Rizzuto R: A forty-kilodalton protein of the inner membrane is the mitochondrial calcium uniporter. Nature 476: 336-340, 2011.

30. Baughman JM, Perocchi F, Girgis HS, et al: Integrative genomics identifies MCU as an essential component of the mitochondrial calcium uniporter. Nature 476: 341-345, 2011.

31. Dash RK and Beard DA: Analysis of cardiac mitochondrial $\mathrm{Na}^{+} / \mathrm{Ca}^{2+}$ exchanger kinetics with a biophysical model of mitochondrial $\mathrm{Ca}^{2+}$ handling suggests a 3:1 stoichiometry. J Physiol 586: 3267-3285, 2008.

32. Jouaville LS, Pinton P, Bastianutto C, Rutter GA and Rizzuto R: Regulation of mitochondrial ATP synthesis by calcium: evidence for a long-term metabolic priming. Proc Natl Acad Sci USA 96: 13807-13812, 1999.

33. Komary Z, Tretter L and Adam-Vizi V: $\mathrm{H}_{2} \mathrm{O}_{2}$ generation is decrease by calcium in isolated brain mitochondria. Biochim Biophys Acta 1777: 800-807, 2008.

34. Rizzuto R, Marchi S, Bonora M, et al: $\mathrm{Ca}^{2+}$ transfer from the ER to mitochondria: when, how and why. Biochim Biophys Acta 1787: 1342-1351, 2009.

35. Krause SM, Jacobus WE and Becker LC: Alterations in cardiac sarcoplasmic reticulum calcium transport in the postischaemic 'stunned' myocardium. Circ Res 65: 526-530, 1989.
36. Basso E, Fante L, Fowlkes J, Petronilli V, Forte MA and Bernardi P: Properties of the permeability transition pore in mitochondria devoid of Cyclophilin D. J Biol Chem 280: 18558-18561, 2005.

37. Regula KM and Kirshenbaum LA: Apoptosis of ventricular myocytes: a means to an end. J Mol Cell Cardiol 38: 3-13, 2005.

38. Starkov AA, Chinopoulos C and Fiskum G: Mitochondrial calcium and oxidative stress as mediators of ischemic brain injury. Cell Calcium 36: 257-264, 2004.

39. Halestrap A: Biochemistry: a pore way to die. Nature 434: 578-579, 2005.

40. Hardy L, Clark JB, Usmar VM, DR and Stone D: Reoxygenation-dependent decrease in mitochondrial NADH: CoQ reductase (complex I) activity in the hypoxic/reoxygenated rat heart. Biochem J 274: 133-137, 1991.

41. Sousa SC, Maciel EN, Vercesi AE and Castilho RF: $\mathrm{Ca}^{2+}$-induced oxidative stress in brain mitochondria treated with the respiratory chain inhibitor rotenone. FEBS Lett 543: 179-183, 2003.

42. Yadava N and Nicholls DG: Spare respiratory capacity rather than oxidative stress regulates glutamate excitotoxicity after partial respiratory inhibition of mitochondrial complex I with rotenone. J Neurosci 27: 7310-7317, 2007.

43. Grijalba M,Vercesi A and Schreier S: $\mathrm{Ca}^{2+}$-induced increased lipid packing and domain formation in submitochondrial particles. A possible early step in the mechanism of $\mathrm{Ca}^{2+}$-stimulation generation of reactive oxygen species by the respiratory chain. Biochemistry 38: 13279-13287, 1999.

44. Gincel D, Zaid H and Shoshan-Barmatz V: Calcium binding and translocation by the voltage-dependent anion channel: a possible regulatory mechanism in mitochondrial function. Biochem J 358: 147-155, 2001.

45. Wikström M and Saari H: A spectral shift in cytochrome a induced by calcium ions. Biochim Biophys Acta 408: 170-179, 1975.

46. Cleeter MW, Cooper JM, Darley-Usmar VM, Moncada S and Schapira AH: Reversible inhibition of cytochrome c oxidase, the terminal enzyme of the mitochondrial respiratory chain, by nitric oxide. Implications for neurodegenerative diseases. FEBS Lett 345: 50-54, 1994.

47. Votyakova TV and Reynolds IJ: $\mathrm{Ca}^{2+}$-induced permeabilization promotes free radical release from rat brain mitochondria with partially inhibited complex I. J Neurochem 93: 526-537, 2005.

48. Vygodina TV, Dyuba AV and Konstantinov AA: Effect of calcium ions on electron transfer between hemes a and a(3) in cytochrome c oxidase. Biochemistry 77: 901-909, 2012.

49. Tretter L, Biagioni Angeli E, Ardestani MR, Goracci G and Adam-Vizi V: Reversible inhibition of hydrogen peroxide elimination by calcium in brain mitochondria. J Neurosci Res 89: 1965-1972, 2011.

50. Zorov DB, Juhaszova M and Sollott SJ: Mitochondrial ROS-induced ROS release: an update and review. Biochim Biophys Acta 1757: 509-517, 2006.

51. Ly JD, Grubb DR and Lawen A: The mitochondrial membrane potential $\left(\Delta \Psi_{\mathrm{m}}\right)$ in apoptosis; an update. Apoptosis 8: 115-128, 2003. 